\title{
Severe migraine-like headache as unique symptom of steroid withdrawal syndrome betamethasone-induced in lumbar disc herniation
}

\author{
Domenico Chirchiglia', Pasquale Chirchiglia', Giulio Di Mizio², Luca Gallelli³ \\ ${ }^{1}$ Department Of Neurosurgery, University Of Catanzaro, Italy \\ ${ }^{2}$ Department Of Forensic Sciences, University Of Catanzaro, Italy \\ ${ }^{3}$ Department Of Pharmacology, University Of Catanzaro, Italy
}

\begin{abstract}
Background. We describe the unusual case of a person suffering from lumbar pain, by herniated disc, treated with betamethasone. The abrupt suspension of the drug caused so called steroid withdrawal syndrome that may occur when receiving a glucocorticoid hormone for a long time it is abruptly stopped.

Method. We administered it to a patient suffering from intense lumbar pain. The drug was suspended abruptly a few days after the onset of therapy.

Results. The reaction to the suspension of the steroid was the onset of a severe migraine headache, which disappeared with the re-administration of betamethasone.

Conclusions. What are the causal hypotheses of this event? An increase in endocranial pressure, of pseudotumor cerebri type? A case of delayed hypersensitivity, since the subject had already taken on betamethasone, without reporting any side effects? We think that the disorder is due to a condition of late hypersensitivity to the steroids, from unknown cause.
\end{abstract}

Keywords: betamethasone, corticoid withdrawal syndrome, sensitization

\section{INTRODUCTION}

Steroid withdrawal syndrome (SWS) is a condition that can occur when a patient stops abruptly corticoid medications.

Symptoms are the following: joint pain, a sensation of discomfort, inflammation, soreness, achiness, or stiffness in a joint or joints of the body, anorexia known as a lack of or loss of appetite for food, vomiting, cravings, desires for unusual foods. irritability, an emotional behavior that is result of feelings of frustration and/or annoyance. Adrenal insufficiency is responsible for SWS (1). It is a temporary form of secondary adrenal insufficiency that may occur when a person who hired a glucocorticoid hormone for a long time abruptly stops or interrupts the medication. Glucocorticoid hormones, which are often used to treat inflammatory illnesses like rheumatoid arthritis, asthma, or ulcerative colitis, block the release of both corticotropin-releasing hormone (CRH) and adreno -corticotropic hormone ( ACTH ). Normally, CRH instructs the pituitary gland to release ACTH. If CRH levels drop, the pituitary is not stimulated to release ACTH, and the adrenals then fail to secrete sufficient levels of cortisol. Prednisolone is the most involved steroid in steroid withdrawal syndrome. The type of treatment is related to the re-administration of the cortisone concerned (2).

In the course of therapy with steroids, especially for intensive and prolonged treatments, the most frequent adverse effects are: decreased tolerability to carbohydrates and possible manifestation of latent diabetes mellitus, cutaneous alterations such as delays in the processes of scarring, and fragility of 
the skin, depressive symptoms. Headache is rarely reported, except if it is pre-existing (3).

In this study we describe the unusual case of a person suffering from lumbar pain, by herniated disc, treated with betamethasone. The abrupt suspension of the drug caused severe migraine headache, that disappeared with the re-administration of betamethasone.

\section{MATERIAL AND METHODS}

The case concerns a 43 year-old male suffering from low back pain, caused by herniated disc, irradiating along the right lower limb, severe intensity, associated with paresthesias and difficult gait. A lumbar CT showed a small right L4-L5 disc erniation.He was given the following treatment: diclofenac sodium $75 \mathrm{mg}$ intramuscular vial, one per day, thiocolchicoside $4 \mathrm{mg}$ intramuscular vial, one per day, betamethasone injection $4 \mathrm{mg}$, one vial per day. After 3 days there was a slight improvement on pain, followed by a further improvement on the seventh day. The patient suspended abruptly betamethasone, assuming only diclofenac and thiocolchicoside. Two days after discontinuation of betamethasone, he presented the sudden onset of a headache with features of severe migraine. The pain was unilateral, pulsating, unbearable, forcing the patient to bed, unable to do anything. Vomiting, phono-photofobia and tachycardia were associated symptoms. The patient was not suffering from migraine, nor headache. After a day the patient was hospitalized for the persistence of headache. A brain CT was performed, resulting normal as well as laboratory tests. The patient continued to assume diclofenac and thiocolchicoside but the low back pain did not improve. Betamethasone was assumed again, $4 \mathrm{mg}$ one intramuscular vial per day and headache disappeared in one day. After 4 days, the patient began the suspension of betamethasone which was taken every other day for a week, until the final suspension.

\section{RESULTS}

Headache disappeared completely and also the other two drugs were suspended. Lower back pain improved completely. To point out that the patient did not suffer from migraine and other occasions took both anti-inflammatory drugs and betamethasone, reporting that in other episodes betamethasone was reduced gradually and no headache was reported.

\section{DISCUSSION}

Steroids withdrawal syndrome (SWS) is an acute condition due to chronic intake and also to the dose taken of such drugs when they are interrupted abruptly. Corticosteroids, also called adrenocortical hormones or corticoids, are a group of steroid hormones synthesized in the adrenal cortex. According to their physiological function, they are divided into three groups, the glucocorticoids, so called for their importance in the metabolism of the glucose, the mineralocorticoids, active in the balance of the mineral salts, in particular of the sodium and potassium and sex hormones (4).

Glucocorticoids - Cortisol is without a doubt the most known glucocorticoid, although several hormones are included in the same family, such as cortisone and corticosterone. All these glucocorticoids act to raise blood glucose, increasing the production of glucose from some amino- acids and decreasing its peripheral use. These hormones also stimulate the storage of glycogen in the liver. The increase in blood glucose concentration is important to ensure normal brain activity under stressful conditions.

Furthermore, corticoid hormones have a catabolic effect (decrease bone mineralization, promote protein catabolism and stimulate the mobilization of fatty acids and glycerol from adipose deposits) (4).

Glucocorticoids carry out an important activity on the immune system and have, on the whole, a soppressive action on the body defenses. This property is abundantly used in therapy to reduce inflammatory states (traumatic, asthmatic, arthritic, rheumatic, allergic).

Unfortunately, the use of such drugs presents a long series of side effects. For this reason, they should be administered extrema ratio, when the disease does not benefit from other medications, always taking into account that their suspension should be gradual, to avoid the withdrawal syndrome.

Betamethasone is a steroidal anti-inflammatory drug, belonging to the class of long-acting gluco- 
corticoids. Side effects are very rare if the drug is used for short periods (less than 7 days) or for topical use, since the absorption through the skin is minimal (about $0.2 \%$, with variations depending on the body district). However, prolonged treatments can cause diabetes mellitus, Cushing iatrogenic syndrome, gastric ulcer, sodium retention, and the potassium leakage, cataract, increased intraocular pressure with possible onset of glaucoma, depression. Dexamethasone and Betamethasone are the most closely involved corticosteroids in the withdrawal syndrome. The reason is the active ingredient of the medications, the duration of the treatment and the cumulative dose. However, some individuals who take dexamethasone and betamethasone at low doses and for periods not exceeding 10 days may inhibit the hypothalamus-pituitary axis and promote withdrawal syndrome(5). In lumbar pain and sciatica, betamethasone is widely used. The side effects, in non-prolonged use (around a week) are very rare $(4,6)$. Headache is much rarer, in particular migraine-like headache. In the case we described, two events have attracted our attention: first, the appearance of drug withdrawal syndrome, after a few days of treatment, second, the appearance of severe migraine-like headache, an unusual side effect, in a person who is not suffering from headache or even less migraine. The mechanism that caused such a severe headache could be explained by an increase in intracranial pressure, caused by the steroid, through the effect on hydro-saline metabolism, causing fluid retention $(7,8)$.

Secondary adrenal insufficiency following withdrawal of corticosteroids has been found in $20.5 \%$ of patients after stopping them(9). The association is highest with higher doses and duration of treatment. The temporal association between the cessation of patients's corticosteroids and onset of headache suggests secondary adrenal insufficiency as the cause of intracranial hypertension $(10,11)$. The gradual improvement of symptoms following steroid replacement would support further this hypothesis. SWS is linked to sensitization by medications, a process in which repeated administration of a stimulus results in the progressive amplification of a response. Sensitization is due to alteration of the receptors of neurotransmitters such as dopamine and serotonin $(12,13)$, to which the drug is lacking. Sensitization is a both adaptive and maladaptive process when the organism is sensitized with harmful stimuli.

\section{CONCLUSIONS}

Corticoid withdrawal syndrome is an infrequent condition, affecting people who stop medications abruptly. This can cause a series of symptoms, sometimes severe. We describe a case of abrupt betamethasone withdrawal, in a patient suffering from low back pain, manifesting with severe migraine headache, that is a very rare symptom, especially because it occurred alone, not followed by other symptoms. The subject had already taken betamethasone in the past, without manifesting adverse effects. Moreover, although abrupt, suspension should not trigger a withdrawal syndrome after a short treatment. We think that the disorder is due to a condition of late hypersensitivity to the steroids, from unknown cause.

\section{Conflict of interest: none declared Financial support: none declared}

\section{REFERENCES}

1. Fourie DT. Recovery of steroid induced adrenal insufficiency. $S$ Afr Med J. 1977 Aug 13;52(8):301.

2. Yates M, Watts RA, Swords F, MacGregor AJ. Glucocorticoid withdrawal in polymyalgia rheumatica: the theory versus the practice. Clin Exp Rheumatol. 2017 Jan-Feb;35(1):1-2. Epub 2017 Jan 5.

3. Cevoli S, Giannini G, Favoni V2,3, Terlizzi R2, Sancisi E, Nicodemo M, Zanigni S, Bacchi Reggiani ML, Pierangeli G, Cortelli P. Treatment of withdrawal headache in patients with medication overuse headache: A pilot study. J Headache Pain. 2017 Dec;18(1):56. doi: 10.1186/s10194-017-0763-9. Epub 2017 May 12.

4. Dixon RB, Christy NP. On the various forms of corticosteroid withdrawal syndrome. Am J Med. 1980 Feb;68(2):224-30.

5. Chabre 01, Goichot B2, Zenaty D3, Bertherat J4. Group 1. Epidemiology of primary and secondary adrenal insufficiency:

Prevalence and incidence, acute adrenal insufficiency, long-term morbidity and mortality. Ann Endocrinol (Paris). 2017 Dec;78(6):490494. doi: 10.1016/j.ando.2017.10.010. Epub 2017 Nov 27.

6. Quinkler M, Ekman B, Marelli C, Uddin S, Zelissen P, Murray RD; EU-AIR Investigators. Prednisolone is associated with a worse lipid profile than hydrocortisone in patients with adrenal insufficiency. Endocr Connect. 2017 Jan;6(1):1-8. Epub 2016 Nov 18.

7. Rushworth RL, Chrisp GL, Torpy DJ. Pseudotumor cerebri following oral betamethasone for common cold. Endocr Pract. 2018 Mar 2. doi: 10.4158/EP-2017-0117.

8. Balasubramanian S, Shivbalan S, Ganesh R. Benign intracranial hypertension after topical steroid withdrawal. Indian J Pediatr. 2005 Nov; 72(11):994. 
9. Younes AK, Younes NK. Recovery of steroid induced adrenal insufficiency. Transl Pediatr. 2017 Oct;6(4):269-273. doi: 10.21037/ tp.2017.10.01. Review.

10. Hedner P, Kullberg G, Bostedt I. Recovery of the hypothalamus-pituitary-adrenal axis after short term high dose corticosteroid treatment in neurosurgical practice. Acta Neurochir (Wien). 1984;73(3-4):157-63.

11. Sandra Shenouda, Khaled Al-Farawi, Jenna Dolan, Susan L Flesher. Idiopathic intracranial hypertension as a presenting sign of adrenal insufficiency. SAGE Open Med Case Rep. 2018; 6: $2050313 \times 17753787$.

12. Corazza M, Mantovani L, Maranini C, Bacilieri S, Virgili A. Contact sensitization to corticosteroids: increased risk in long term dermatoses. Eur J Dermatol. 2000 Oct-Nov; 10(7):533-5.

13. Wilkinson SM1. Hypersensitivity to topical corticosteroids. Clin Exp Dermatol. 1994 Jan;19(1):1-11. 